RESEARCH PAPER

\title{
White matter connections of the supplementary motor area in humans
}

\author{
Francesco Vergani, ${ }^{1,2}$ Luis Lacerda, ${ }^{3}$ Juan Martino, ${ }^{4}$ Johannes Attems, ${ }^{5}$ \\ Christopher Morris, ${ }^{2,6}$ Patrick Mitchell, ${ }^{1,2}$ Michel Thiebaut de Schotten, ${ }^{7,8}$ \\ Flavio Dell'Acqua ${ }^{3,9}$
}

For numbered affiliations see end of article.

\section{Correspondence to} Dr Francesco Vergani, Department of Neurosurgery, Regional Neurosciences Centre Royal Victoria Infirmary, Queen Victoria road, Newcastle upon Tyne NE1 4LP, UK; francesco.vergani@ncl.ac.uk

FV and LL contributed equally to this study.

Received 20 December 2013 Revised 18 March 2014 Accepted 24 March 2014 Published Online First 16 April 2014

CrossMark

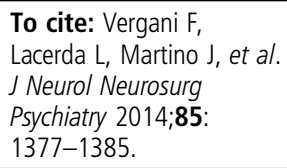

\begin{abstract}
Introduction The supplementary motor area (SMA) is frequently involved by brain tumours (particularly WHO grade II gliomas). Surgery in this area can be followed by the 'SMA syndrome', characterised by contralateral akinesia and mutism. Knowledge of the connections of the SMA can provide new insights on the genesis of the SMA syndrome, and a better understanding of the challenges related to operating in this region.

Methods White matter connections of the SMA were studied with both postmortem dissection and advance diffusion imaging tractography. Postmortem dissections were performed according to the Klingler technique. 12 specimens were fixed in $10 \%$ formalin and frozen at $-15^{\circ} \mathrm{C}$ for 2 weeks. After thawing, dissection was performed with blunt dissectors. For diffusion tractography, high-resolution diffusion imaging datasets from 10 adult healthy controls from the Human Connectome Project database were used. Whole brain tractography was performed using a spherical deconvolution approach.
\end{abstract}

Results Five main connections were identified in both postmortem dissections and tractography reconstructions: (1) U-fibres running in the precentral sulcus, connecting the precentral gyrus and the SMA; (2) U-fibres running in the cingulate sulcus, connecting the SMA with the cingulate gyrus; (3) frontal 'aslant' fascicle, directly connecting the SMA with the pars opercularis of the inferior frontal gyrus; (4) medial fibres connecting the SMA with the striatum; and (5) SMA callosal fibres. Good concordance was observed between postmortem dissections and diffusion tractography.

Conclusions The SMA shows a wide range of white matter connections with motor, language and lymbic areas. Features of the SMA syndrome (akinesia and mutism) can be better understood on the basis of these findings.

\section{INTRODUCTION}

The supplementary motor area (SMA) is an eloquent region of the brain that plays an important role in the planning, initiation and learning of complex motor functions. ${ }^{1-4}$ The SMA in the dominant hemisphere is also involved in the initiation of speech. ${ }^{5}$ From a surgical perspective, the SMA is important as it is frequently involved by brain tumours; up to $10 \%$ of de novo glioblastomas and $27 \%$ of WHO grade II gliomas occur in this region. $^{7}$ It is also a frequent surgical target for drug-resistant frontal lobe epilepsy. ${ }^{8}$ Surgery in this area can be followed by the 'SMA syndrome', as originally described by Laplane, ${ }^{9}$ characterised by contralateral akinesia and, for surgery in the dominant hemisphere, mutism or speech reduction (also referred to as 'transcortical motor aphasia'). These symptoms tend to resolve over time, but can affect patients for several months and can occasionally be permanent. $^{61011}$ Knowledge of the connections of the SMA can help to understand the pathogenesis of the SMA syndrome, providing the neurosurgeon with a better understanding of the challenges related to operating in this region, as well as offering patients a more accurate preoperative counselling.

Although the connections of the SMA have been extensively studied in the primate with a detailed analysis of the connections of the pre-SMA and SMA-proper, ${ }^{12}{ }^{13}$ limited data exist about the SMA connectivity in humans. The common method used to investigate the white matter in the primate is the autoradiography technique, where a neuronal tracer is injected in the area of interest and the connections of that particular area are then established with postmortem histology. ${ }^{14}$ In humans, two techniques are available to investigate white matter anatomy: postmortem dissection and diffusion tractography. Dissection of cadaveric specimens has been used for centuries to study the anatomy of the brain, including the tracts of white matter. ${ }^{15}$ In recent years, the technique originally described by Klingler ${ }^{16}$ has been revitalised in the neurosurgical community. ${ }^{17}$ A modification of this technique has been recently suggested, with the aim of sparing the cortex during the dissection (the so-called 'cortex-sparing' dissection). ${ }^{18}$ The advantage is maintaining the 3D relationship between the fibre tracts and their cortical terminations, thus avoiding the loss of fundamental landmarks during the dissection. Diffusor tractography is a relatively new MRI technique, and it is based on the principle that the diffusion of water molecules in white matter tracts is anisotropic, with the highest diffusivity occurring along the direction of fibres. Using mathematical models it is possible to estimate the one or more fibre orientations in each voxel and use this information to reconstruct the main white matter pathways with the unique advantage of investigating the white matter human anatomy in vivo. $^{19}$

Diffusion tensor imaging and more advanced diffusion imaging methods have been used to study in humans the connectivity of the SMA region, showing fibres connecting the SMA with the fronto-opercular region (area 44 or 'Broca's area') 
and the striatum. ${ }^{20-23}$ However, there is no study in the literature that has comprehensively reviewed the white matter fibres connecting the SMA and no study using postmortem dissections has been dedicated to investigate this aspect.

In this study, for the first time we present a combined approach to investigate the SMA connections, with postmortem dissections and diffusion imaging performed in vivo on healthy volunteers. Original anatomical data about the SMA white matter connections are presented and discussed.

\section{METHODS}

\section{Postmortem dissections}

Postmortem dissection of white matter fibres was performed according to the technique originally described by Klingler. ${ }^{16}$ Twelve hemispheres in total were used for the present study (six right, six left). The hemispheres were collected at the Newcastle Brain Tissue Resource (Institute for Ageing and Health, Newcastle University, Newcastle upon Tyne, UK) and at the Department of Anatomy of Cantabria University (Santander, Spain). Both Institutions have ethical approval to use postmortem human specimens for research purpose. The specimens were fixed in $10 \%$ formalin solution for a minimum of 3 months. After removal of the pia-arachnoid membrane and cortical vessels, the hemispheres were frozen at $-15^{\circ} \mathrm{C}$ for 15 days. The water crystallisation induced by the freezing process disrupts the structure of the grey matter (which has a high water content), thus making it easier to peel off the cortex from the underlying white matter. The freezing process also separates the white matter fibres, facilitating the dissection of the tracts. The specimens were washed under running water for several hours before performing the dissection.

The superficial anatomy of each hemisphere was studied in detail, with identification of the sulci and gyri. The dissection was then started, with removal of the cortex and exposure of the underlying U-fibres (also known as intergyral or arcuate fibres). The white matter dissection was then completed in a stepwise manner, from lateral to medial and from medial to lateral. Wooden spatulas were used in the initial step of the dissection to carefully remove the cortex. Once the U-fibres were identified, the dissection was performed using blunt metallic dissectors with different tip sizes. Care was taken to separate the fibres using the blunt edge of the instrument, thus avoiding the generation of spurious tracts. In addition, dissection was performed according to the 'cortex-sparing' technique recently described by Martino et al. ${ }^{18}$ According to this technique, as much as possible of the cortex is preserved during the dissection in order to maintain the 3D relationship between the fibres and their cortical terminations. Digital images were acquired during the dissection.

\section{MRI data}

High-resolution diffusion imaging datasets from 10 adult healthy controls from the Human Connectome Project (HCP) database (http://www.humanconnectome.org, Release Q2) were used for this study.

Briefly, datasets were acquired with a voxel size of $1.25 \times 1.25 \times 1.25 \mathrm{~mm}$, field of view $=210 \times 210 \mathrm{~mm}, 111$ slices, $\mathrm{TE}=89.50 \mathrm{~ms}, \mathrm{TR}=5520 \mathrm{~ms}, \mathrm{~b}-\mathrm{value}=1000,2000,3000 \mathrm{~s} /$ $\mathrm{mm}^{2}, 90$ directions per $\mathrm{b}$-values and 18 non-diffusion-weighted volumes. In this study, only data with a b-value of $2000 \mathrm{~s} / \mathrm{mm}^{2}$ were used. Data were preprocessed using the default HCP preprocessing pipeline (V.2), which includes correction for susceptibility, motion and eddy current distortions. Spherical deconvolution was then performed using the damped
Richardson-Lucy deconvolution algorithm. ${ }^{24}$ Algorithm parameters were $\alpha=1.5$, algorithm iteration $=400$ and $\eta=0.06$ and $v=8$ as regularisation terms. Spherical deconvolution tractography was performed using a modified Euler tractography algorithm ${ }^{25}$ with a $45^{\circ}$ angle threshold, an absolute hindrance modulated (HMOA) threshold of 0.012 and relative threshold $7 \%$.

After whole brain tractography, semiautomatic dissections of tracts were performed according to predefined cortical and subcortical regions. Starting from an SMA region predefined on the Montreal Neurological Institute (MNI) space, non-linear transformations were calculated from MNI to each subject native space and applied on the SMA region. Other regions of interests were already available in the subject space as part of the HCP processing pipeline and derived from the structural data of each subject using freesurfer parcellation. Where required, exclusion regions of interest were manually placed to exclude remaining spurious or non-anatomically consistent streamlines. For each tract, binary maps were generated to identify voxels visited by at least one streamline and then averaged in the MNI space to generate visitation maps for each tract. In this study, we investigated for both hemisphere connections among the SMA and the inferior frontal gyrus (IFG), Caudate nucleus, precentral gyrus (PreCG) and cingulate gyrus.

Tract volume was used to estimate the lateralisation index (LI) for each tract using the following formula: $\mathrm{LI}=$ (Tract Volume left - Tract Volume right)/((Tract Volume left+Tract Volume right)/2). Positive values indicate a left lateralisation. For each tract, a one sample t-test on the LI was performed using Bonferroni correction with an adjusted $\alpha$ level of 0.0125 per test (ie, 0.05/4). A Shapiro-Wilk test was used to verify normal distribution of LI in all tracts.

\section{RESULTS}

Each specimen was accurately examined and the major sulci and circonvolutions were identified. The central sulcus, dividing the PreCG and postcentral gyri, was the initial landmark to be identified on the lateral convexity. The precentral sulcus, anterior to the PreCG, was used as the posterior border of the SMA. The superior frontal sulcus, separating the superior frontal gyrus (SFG) and middle frontal gyrus (MFG), was considered as the infero-lateral border of the SMA. ${ }^{26} 27$ On the medial aspect of the brain, the cingulate sulcus, separating the mesial aspect of the SFG and the cingulate gyrus, represented the infero-medial border of the SMA. The anterior border was considered to be $5 \mathrm{~cm}$ in front of the precentral sulcus. The SMA is divided in pre-SMA and SMA-proper by a vertical line passing through the anterior commissure (vertical commissure anterior (VCA) line). On the medial aspect of each hemisphere, the VCA line was traced and the two regions of the SMA were identified (figure 1).

\section{Dissection of the lateral aspect of the SMA Short U-fibres}

After removal of the cortex, the first fibres to be exposed on the convexity of the hemisphere were short arcuate fibres connecting neighbouring gyri (also known as 'U-fibres'). In particular, U-fibres were encountered in the depth of the precentral sulcus, connecting the posterior part of the SMA-proper with the PreCG. The number and distribution of these fibres appeared to be variable, but a short arcuate fibre was always present in close proximity of the 'hand knob' of the primary motor cortex. This is a 'knob-like' portion of the PreCG corresponding to the middle knee of the central sulcus and representing the anatomical localisation of the motor hand area. Tractography showed consistently these connections bilaterally but without any 


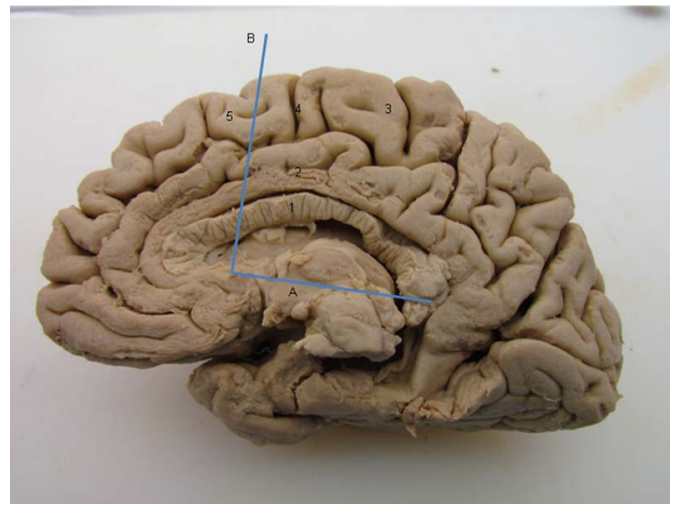

Figure 1 Medial aspect of a right hemisphere. The anterior commissure - posterior commissure (AC-PC) line is demonstrated (A). The VCA line (B) perpendicular to the AC-PC line at the level of the anterior commissure divides the pre-SMA anteriorly (5) from the SMA-proper (4). The corpus callosum (1), cingulate gyrus (2) and central lobule (3) are also indicated. SMA, supplementary motor area.

lateralisation $(\mathrm{LI}=-0.13 \pm 0.34, \mathrm{p} \quad$ value $=0.27)$. Similarly, U-fibres were encountered in the depth of the superior frontal sulcus, connecting the lateral aspect of the SMA with the MFG (figure 2, table 1).

Figure 2 (A) Lateral dissection of the SMA (right hemisphere). U-fibres are demonstrated in the depth of the precentral sulcus, connecting the PreCG and SMA-proper (red pins). U-fibres are also present in the superior frontal sulcus, connecting the SMA with the MFG (green pins).

(1) PoCG; (2) PreCG; (3) SMA-proper; (4) SFG; and (5) MFG. (B) Diffusion tractography demonstrating the right and left connections of the SMA with the PreCG. Note the U-shaped fibres linking the two gyri. SMA, supplementary motor area; SFG, superior frontal gyrus; MFG, middle frontal gyrus; PreCG, precentral gyrus; PoCG, postcentral gyrus.

\section{Frontal aslant tract}

Continuing the dissection of the lateral aspect of the frontal lobe, a direct fibre tract running from the SFG to the pars opercularis of the IFG (area 44, according to Brodmann's classification) was demonstrated. After removal of the MFG and partial removal of the IFG, longitudinal fibres of the superior longitudinal fasciculus (SLF) were exposed. Deeper to the SLF, a vertical bundle of fibres, running from the SMA to the pars opercularis of the IFG, was observed. This fascicle presented an oblique course between the SFG and IFG, and corresponded to the frontal aslant tract (FAT). The cortical termination of this tract in the SFG was at the boundary between the pre-SMA and SMA-proper (figure 3). The tract was clearly demonstrated also with diffusion tractography where a large number of streamlines projected to the IFG pars opercularis. Fewer projections also reached the pars triangularis of the IFG and the inferior PreCG. The tract was always visible on both hemispheres and showed a statically significant left lateralisation $(\mathrm{LI}=0.38 \pm 0.31, \mathrm{p}=0.003)$ (figure 3 , table 1 ).

\section{Dissection of the medial aspect of the SMA}

\section{Short U-fibres}

On the medial aspect of the hemisphere, another set of U-fibres was observed, running in the depth of the cingulate sulcus. These fibres connected the SMA to the cingulate gyrus, running 
Table 1 Tract volume and lateralisation index (LI) for the diffusion tractography results

\begin{tabular}{|c|c|c|c|c|}
\hline & Left volume $(\mathrm{ml})$ & Right volume (ml) & Laterality index & p Value \\
\hline Precentral gyrus & $6.51 \pm 3.63$ & $7.11 \pm 3.75$ & $-0.13 \pm 0.34$ & 0.270 \\
\hline Inferior frontal gyrus-pars opercularis & $7.93 \pm 3.77$ & $5.64 \pm 3.66$ & $0.38 \pm 0.31$ & 0.003 \\
\hline Cingulate gyrus-anterior & $3.25 \pm 1.00$ & $2.98 \pm 1.34$ & $0.10 \pm 0.54$ & 0.562 \\
\hline Caudate & $2.01 \pm 1.95$ & $1.38 \pm 2.22$ & $0.45 \pm 0.69$ & 0.105 \\
\hline
\end{tabular}

perpendicular to the cingulate sulcus. Diffusion tractography confirmed the presence of extensive and bilateral connections between the SMA and the anterior portion of the cingulate gyrus (figure 4). No significant lateralisation was observed with tractography $(\mathrm{LI}=0.10 \pm 0.54, \mathrm{p}=0.56)($ table 1$)$.

\section{Callosal fibres}

Dissecting the mesial aspect of the brain, after removal of the cingulate gyrus, vertical fibres of the corpus callosum were exposed. These callosal fibres, clearly observed also with diffusion tractography, came from both the pre-SMA and the SMA-proper and converged on the rostrum and anterior part of the body of the corpus callosum (figure 5).

\section{Corona radiata and striatal fibres}

Continuing the dissection from medial to lateral, after complete removal of the cingulate gyrus and partial removal of the corpus callosum, the caudate nucleus was exposed. At this level, two sets of fibres running vertically from both the SMA-proper and pre-SMA were identified. The first group to be encountered belonged to the corona radiata and continued inferiorly, with a slight oblique course, into the internal capsule. More laterally, another group of fibres converged directly onto the head and anterior body of the caudate nucleus. These fibres had a more vertical course and were situated more laterally to the callosal and corona radiata fibres. Diffusion tractography also showed connection between SMA and the head of the caudate nucleus (figure 6). However, the low number of streamlines and the rather disperse organisation of this tract produced a high variability in the volume of this tract. No significant lateralisation was observed $(\mathrm{LI}=0.45 \pm 0.69, \mathrm{p}=0.10)$ (table 1$)$.

\section{DISCUSSION}

To the best of our knowledge, the present study is the first in the literature dedicated to investigate the white matter connections of the SMA using both postmortem dissections (according to the traditional Klingler technique and the 'cortex-sparing' method) and advanced diffusion tractography. Overall, a good concordance was found between the two methods. All the connections described were identified with both techniques, with similar trajectories and directions of the fibre tracts observed. Postmortem dissections provided a more qualitative description of the fibres, being able to clearly identify the relationship of the fibres to other white matter tracts and adjacent cortical

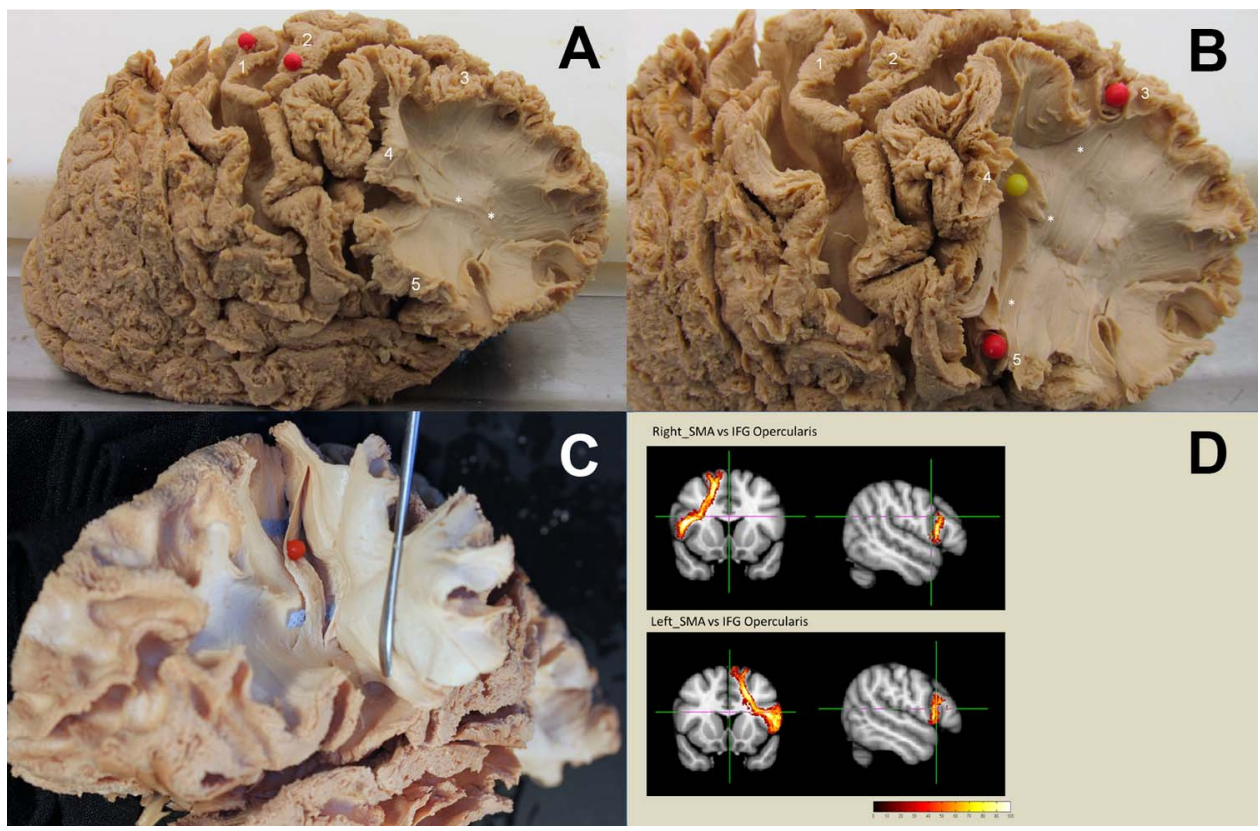

Figure 3 (A) Dissection of lateral aspect of the frontal lobe (right hemisphere): exposure of longitudinally-directed fibres of the SLF (asterisk) after removal of MFG. (1) PoCG; (2) PreCG; (3) SMA; (4) MFG (remnant portion); and (5) IFG. (B) Same specimen. The SLF has been divided (yellow pin), exposing the vertically oriented fibres of the FAT (asterisks), connecting the SMA with the pars opercularis of the IFG (red pins). (1) PoCG; (2) PreCG; (3) SMA; (4) MFG (remnant portion); and (5) IFG. (C) 'Cortex sparing' dissection of the left hemisphere. Exposure of the FAT (red pin). The MFG has been maintained and retracted by a blunt dissector. Note the cortical termination of the aslant tract at the level of the SMA and of the opercular portion of the IFG. (D) Diffusion tractography demonstrating the right and left FAT, connecting the SMA and the IFG. SMA, supplementary motor area; IFG, inferior frontal gyrus; MFG, middle frontal gyrus; SLF, superior longitudinal fasciculus; FAT, frontal aslant tract; PoCG, postcentral gyrus. 


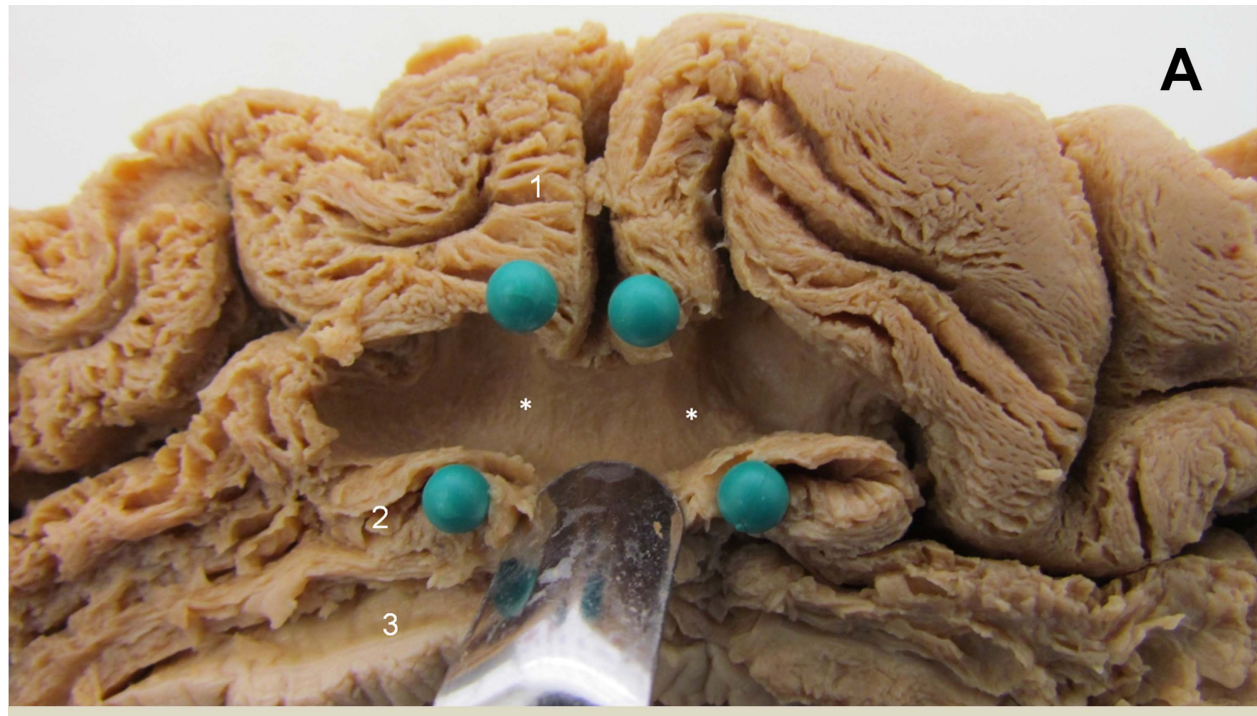

Right_SMA vs Anterior Cingulate

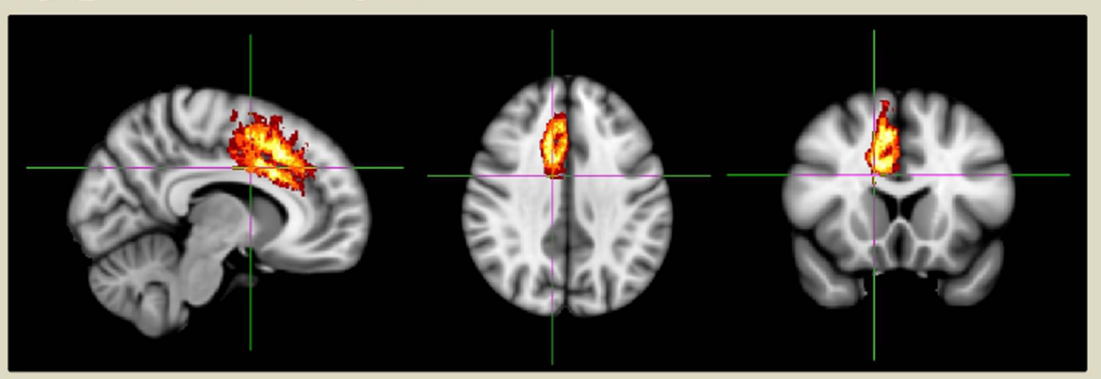

B

Left_SMA vs Anterior Cingulate

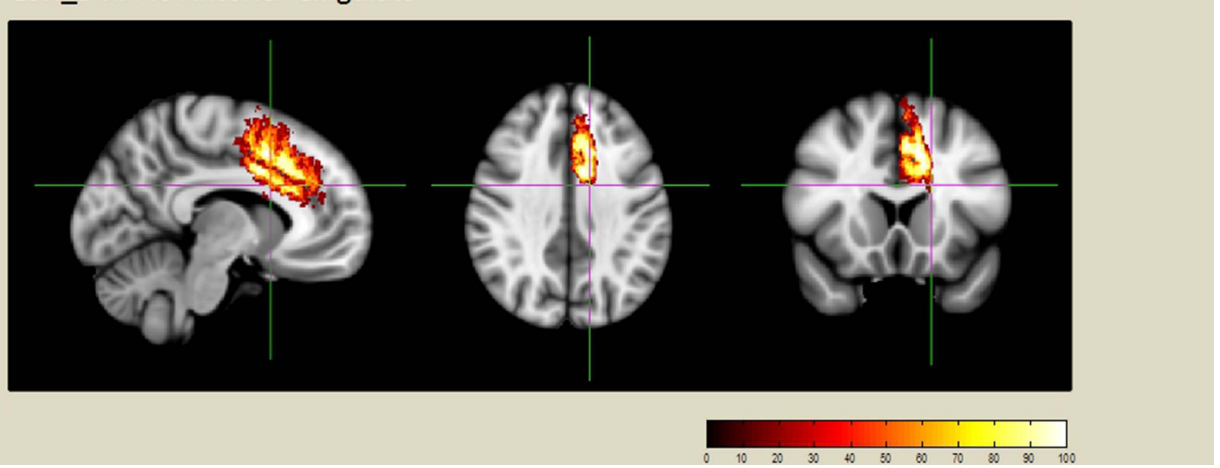

Figure 4 (A) Dissection of mesial aspect of the SMA (right hemisphere). U-fibres running in the cingulate sulcus, connecting the SMA with the cingulate gyrus (asterisks). The green pins show the cortical terminations of these U-fibres. (1) SMA; (2) cyngulate gyrus; and (3) corpus callosum. (B) Diffusion tractography demonstrating the connections between the SMA and the cingulate gyrus. Note the extensive connections with U-fibres to the anterior portion of the cingulate gyrus. SMA, supplementary motor area.

landmarks. Tractography was particularly useful in adding quantitative analysis of tract volumes, making it possible to infer the lateralisation of the tracts described.

\section{SMA and movement}

Direct stimulation of the SMA induces a complex pattern of movements involving different muscles groups. Synergistic movements involving the head, the upper and lower limb (turning the head to look at the hand, grasping movements, etc) have been described. ${ }^{8} 101128$ It is acknowledged that the SMA plays an important role in planning, initiation and execution of movements. In the primate, an SMA-proper and a pre-SMA have been differentiated on the basis of cytoarchitectonic and functional aspects. ${ }^{12}$ Also in humans, in addition to the classic
SMA (SMA-proper), a more rostral area called 'pre-SMA' has been described. Neurophysiological studies suggest that the role of these two regions appear to be different with regard to motor function, with the pre-SMA being involved in higher motor control, such as planning and preparation of movement, while the SMA-proper is more directly related to the execution of movement. ${ }^{2}$ In a recent paper by Ikeda et al, ${ }^{29}$ epicortical field potentials were recorded with subdural electrodes from the pre-SMA and SMA-proper of epileptic patients. Their results showed that the pre-SMA is involved in cognitive motor control and decision making while the SMA-proper is more active in the initiation and execution of voluntary movements. The connections of the two regions can provide an anatomical basis for these functional aspects. It is known from the litearature ${ }^{13}$ that 


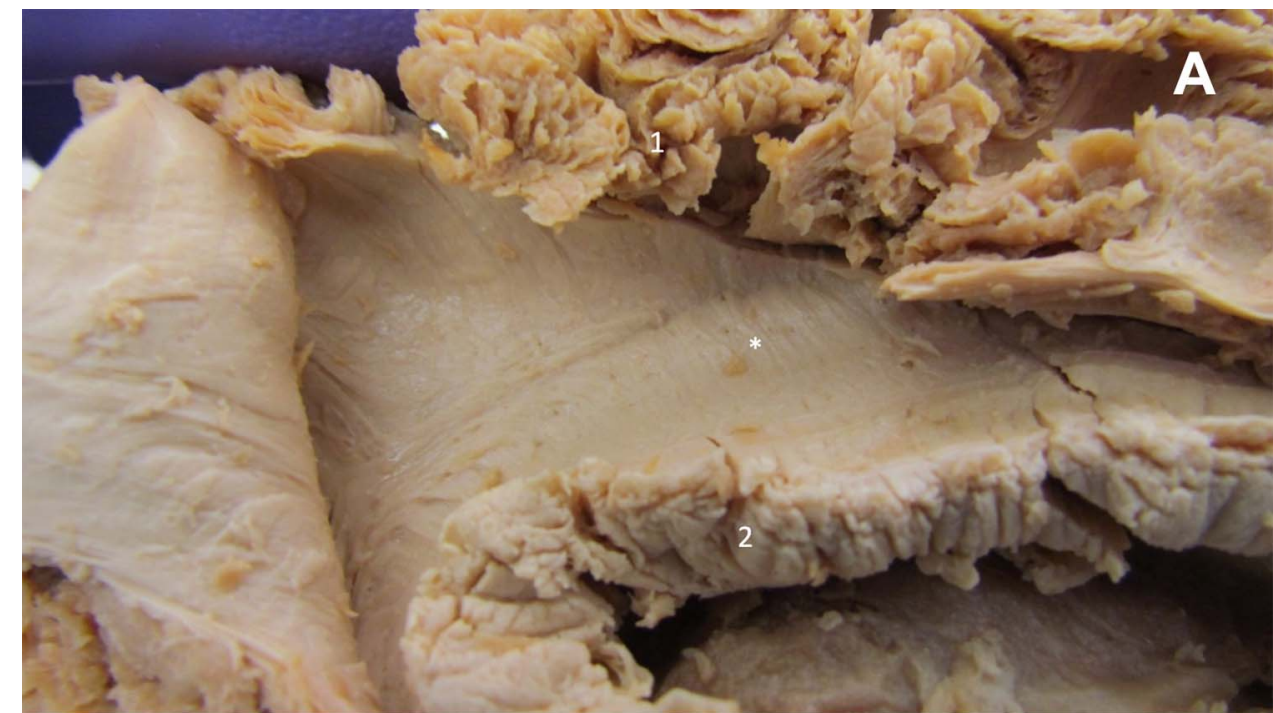

\section{B}

\section{Left_SMA vs Right_SMA}

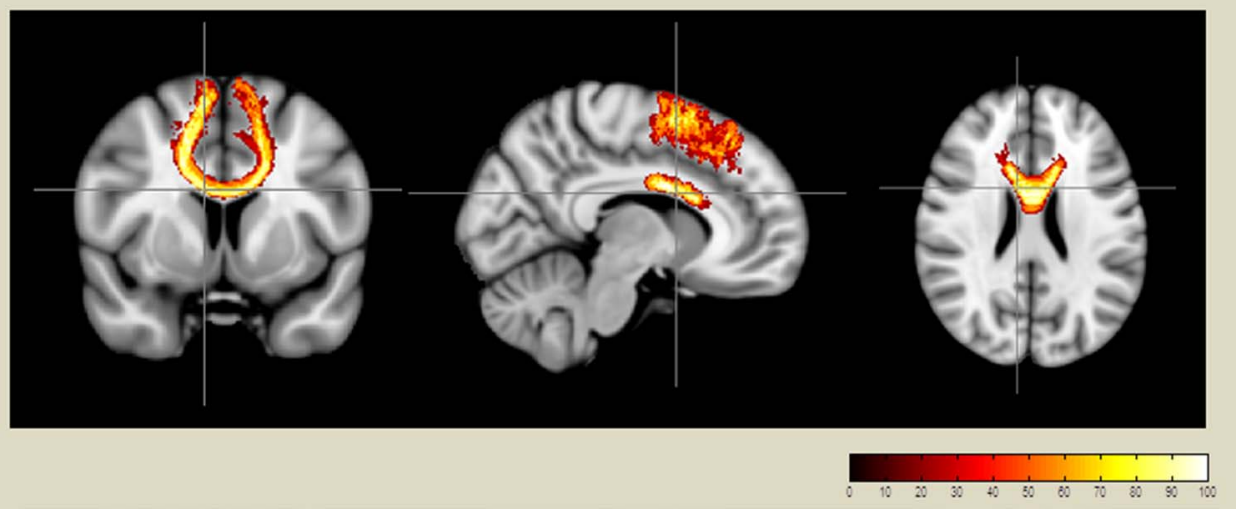

Figure 5 (A) Dissection of mesial aspect of the SMA (right hemisphere). Callosal fibres to the SMA observed after removal of the cingulate gyrus (asterisk). (1) SMA and (2) corpus callosum. (B) Diffusion tractography demonstrating the callosal fibres connecting the left and right SMA. SMA, supplementary motor area.

the SMA-proper sends fibres through the corona radiata and the internal capsule to join the cortico-spinal tract, while the pre-SMA has no direct connection with the spinal cord. Thanks to this cortico-spinal projection, the SMA-proper is thought to play a role in the direct execution of movement. In addition, our study has demonstrated the presence of short U-fibres in the depth of the precentral sulcus, directly connecting the SMA-proper with the primary motor cortex (M1). Of interest, a direct link between the SMA-proper and the M1 appears to be always present at the level of the hand region. It has been hypothesised that the convergence of fibres at this level can play a role in the control of complex hand movements, such as grasping and reaching. ${ }^{30}$ Finally, both the SMA-proper and the pre-SMA have direct connection with the striatum, with fibres directed to the head and body of the caudate nucleus. The presence of striatal connections has been demonstrated in a DTI study by Lehéricy et $a l^{20}$ and the results of our study are in accordance with these previous findings. This cortico-striatal connection is part of a wider network that, through the thalamus, reverberates back to the cortex. This cortico-basal ganglia-thalamo-cortical network is implicated in different aspects of motor control, including initiation, sequencing and modulation of voluntary movements. ${ }^{31}$ The results of our diffusion tractography study suggest a predominance of these fibres on the left side in right-handed individuals.

\section{SMA and language}

Direct stimulation of the dominant SMA has been related to vocalisation and/or arrest of speech. ${ }^{8} 28$ Functional MRI studies have provided useful insights in the role of the SMA in language. The SMA appeared to be activated during different language tasks, such as repetition and silent verbal fluency, with the role of facilitating the initiation of speech. ${ }^{5}{ }^{32}$ In the present study, we demonstrate the presence of a direct connection between the SMA and the pars opercularis of the IFG, corresponding to Broca's area in the dominant hemisphere. This tract has been called 'frontal aslant' (FAT) due to its oblique course. ${ }^{30}$ Our results are in accordance with the previous description of 


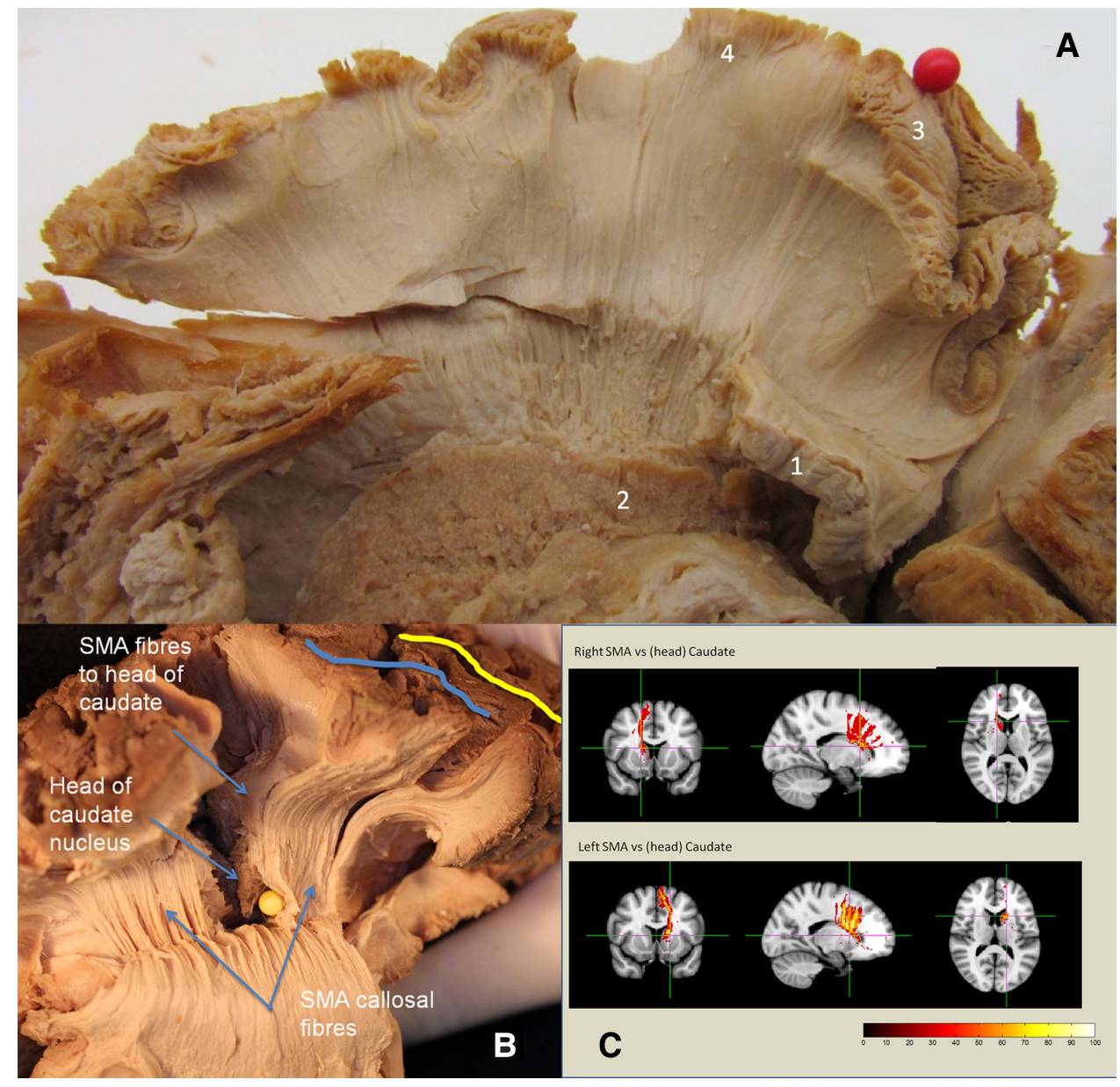

Figure 6 (A) Dissection of mesial aspect of the SMA (right hemisphere). Vertical fibres running from the SMA and converging on the caudate nucleus, seen after complete removal of the cingulate gyrus and of the corpus callosum. Red pin represents the PreCG. (1) Corpus callosum (remnant portion); (2) caudate nucleus; (3) PreCG; and (4) SMA. (B) 'Cortex sparing dissection' of a left hemisphere. The relationship between the SMA fibres to the head of caudate nucleus and the SMA callosal fibres is demonstrated. Yellow pin: ventricle. Blue line: precentral sulcus. Yellow line: central sulcus. (C) Diffusion tractography demonstrating the connections between the SMA and the caudate nucleus (right and left hemisphere). Note the convergence of the fibres onto the head of the caudate. SMA, supplementary motor area; PreCG, precentral gyrus.

the bundle provided by DTI studies. ${ }^{21-23}$ More recently, Kinoshita $e t a l^{33}$ have described a similar bundle connecting the medial frontal cortex and the IFG. The Klingler dissection performed by the authors suggests that the fibres of the FAT merge with the SLF. In our study, the FAT appears to be deeper than the SLF, which had to be cut in order to expose the underlying FAT. The SMA is thought to facilitate speech initiation due to the direct connection to the pars opercularis of the IFG provided by the FAT. In a study of the somatotopy of the SMA, Fontaine et $a l^{10}$ found that the language responses in the SMA were clustered at the border between the pre-SMA and SMA-proper. Interestingly, the border between pre-SMA and SMA-proper represents the cortical termination of the FAT, as demonstrated in the present study by both postmortem dissections and diffusion tractography. To further corroborate the role of the FAT in language, it has been reported that a damage to this tract is associated with decreased verbal fluency in primary progressive aphasia. ${ }^{34}$ Finally, the left lateralisation of the FAT seen in our diffusion tractography, as previously reported by Catani et $a l^{30}$ is also consistent with a possible role in language played by this tract.

In the non-dominant hemisphere it has been suggested that the SMA may mediate voluntary control of face, tongue and pharynx movement through the FAT connecting the SMA and frontal operculum. If the FAT is damaged, the information coming from the SMA cannot reach the oropharyngeal motor cortex, causing a selective volitional palsy as described in a case of Foix-Chavanie-Marie syndrome after removal of a rightsided fronto-insular low grade glioma. ${ }^{35}$

\section{SMA and limbic system}

Studies from the primate have demonstrated extensive connections between the SMA and the limbic system, particularly through fibres directed to the cingulate gyrus. ${ }^{12}{ }^{36}$ Our study confirms the presence of such connections in humans, where several U-fibres were visualised (both on dissection and diffusion tractography), running between the SMA and the cingulate. This direct link between motor and limbic areas is thought to play a role in the motor processing of negative emotional stimuli. ${ }^{37}$ Oliveri et $a l^{38}$ showed that conditioning transcranial magnetic stimulation of the SMA significantly increased the evoked motor potential recorded from the first interosseous muscle during visual-emotional triggered movements (the subjects had to perform movements in response to images with a negative emotional content). The SMA in humans may therefore interface the limbic and the motor systems in the transformation of emotional experiences into motor actions. ${ }^{38}$ The fibres 
connecting the SMA and the cingulate gyrus are one anatomical substrate for this interaction.

White matter connections and the SMA syndrome

Surgery in the SMA can be followed by the 'SMA syndrome', originally described by Laplane in $1977 .{ }^{9}$ Reporting the results of the ablation of the SMA in three patients, Laplane described the clinical course after surgery as being characterised by global akinesia, more prominent contralaterally, with an arrest of speech. This was followed by a phase of recovery, when contralateral reduction of movement and severe reduction of speech were still present. This was followed by a complete recovery, when only a 'disturbance of the alternating movements of the hands"9 was present. Several surgical series related to tumours infiltrating the SMA have confirmed the original observations of Laplane, ${ }^{4} \quad 6 \quad 1011 \quad 27$ usually with more profound and marked symptoms following more extensive resection of the SMA, as it is the case with WHO grade II gliomas. ${ }^{39}$ The outcome is usually favourable, with a complete recovery observed at 612 months after surgery. Minor symptoms, however, can be permanent (underutilisation of contralateral upper limb, mild word finding difficulties, etc). ${ }^{4} 611$

The pathogenesis of the SMA syndrome is not fully understood. Our findings suggest that surgical ablation of the posterior SMA (SMA-proper) is more likely to induce a motor deficit, as this region is strongly connected with motor areas through fibres that directly join the cortico-spinal tract and U-fibres directed to the motor cortex. Specific motor symptoms, like deficit in grasping/reaching, can indeed be explained on the basis of the connection between SMA-proper and the hand region. The wide range of projections from the SMA to the striatum can provide the anatomical basis to explain the reduction in spontaneous movement, which is the hallmark of the SMA syndrome. Akinesia, a cardinal symptom of Parkinsonian disorders, can be the result of damage to the cortico-striatal network in which the SMA plays an important role in facilitating and sequencing voluntary movements. Finally, mutism and language reduction (transcortical motor aphasia), can be explained on the basis of the connection of the SMA with the Broca's area provided by the FAT. Indeed, some features of the classic Broca's aphasia are similar to the mutism observed in the SMA syndrome.

Knowledge of the anatomical connections of the SMA is relevant to the clinical implications of surgery in the SMA region. The degree of cortical and subcortical extension of SMA tumours (as assessed by preoperative MRI and DTI) can help to predict the postoperative course of patients, as previously demonstrated in the case of tumours growing in the ventrolateral frontal region. ${ }^{40}$ This is important to offer patients a more informed counselling on the postoperative course of the SMA syndrome, as well as to plan in advance an individualised rehabilitative intervention.

\section{CONCLUSIONS}

The SMA is at the centre of a rich network of white matter connections with motor, language and lymbic areas. The clinical aspects of the SMA syndrome, including akinesia and mutism, can be better understood on the basis of these anatomical findings.

\section{Author affiliations}

${ }^{1}$ Department of Neurosurgery, Royal Victoria Infirmary, Newcastle upon Tyne, UK ${ }^{2}$ Institute of Neuroscience, Newcastle University, Newcastle upon Tyne, UK

${ }^{3}$ Natbrainlab, Department of Neuroimaging, Institute of Psychiatry, King's College
London, London, UK

${ }^{4}$ Department of Neurological Surgery, Hospital Universitario Marqués de Valdecilla and Instituto de Formación e Investigación Marqués de Valdecilla (IFIMAV),

Santander, Cantabria, Spain

${ }^{5}$ Institute for Ageing and Health, Newcastle University, Newcastle upon Tyne, UK

${ }^{6}$ Medical Toxicology Centre, Newcastle University, Newcastle upon Tyne, UK

${ }^{7}$ Natbrainlab, Department of Forensic and Neurodevelopmental Sciences, Institute of

Psychiatry, King's College London, London, UK

${ }^{8}$ Université Pierre et Marie Curie-Paris 6, Centre de Recherche de I'Institut du Cerveau et de la Moelle épinière (CRICM), UMRS 975, INSERM U 975, CNRS UMR, Paris, France

${ }^{9} \mathrm{NIHR}$ Biomedical Research Centre for Mental Health at South London and Maudsley NHS Foundation Trust and King's College London, Institute of Psychiatry, London, UK

Acknowledgements Specimens for postmortem dissection were provided by the Newcastle Brain Tissue Resource (Institute for Ageing and Health, Newcastle University, Newcastle upon Tyne, UK) and by the Department of Anatomy of Cantabria University (Santander, Spain). Diffusion data were provided by the Human Connectome Project, WU-Minn Consortium (Principal Investigators: David Van Essen and Kamil Ugurbil; 1U54MH091657) funded by the $16 \mathrm{NIH}$ Institutes and Centers that support the NIH Blueprint for Neuroscience Research and by the McDonnell Center for Systems Neuroscience at Washington University.

Contributors All the authors listed have made a substantial contribution to the scientific and intellectual content of the article and take responsibility for it. The first two authors listed (FV and LL) contributed equally to the article.

Funding The Newcastle Brain Tissue Resource is funded in part by a grant from the UK Medical Research Council (G0400074) and by Brains for Dementia research, a joint venture between Alzheimer's Society and Alzheimer's Research UK. JM receives specific funding from the 11/18 API grant entitled "Estudio de la conectividad conectividad cerebral mediante disección de fibras estructural"; "Fundación Marqués de Valdecilla", Instituto de Formación e Investigación Marqués de Valdecilla (IFIMAV), Santander, Cantabria, Spain. 8 October 2010.

Competing interests None.

Provenance and peer review Not commissioned; externally peer reviewed.

\section{REFERENCES}

1 Penfield W, Welch $\mathrm{K}$. The supplementary motor area of the cerebral cortex: a clinical and experimental study. Arch Neurol Psychiatry 1951;66:289-317.

2 Orgogozo JM, Larsen B. Activation of the supplementary motor area during voluntary movement in man suggests it works as a supramotor area. Science 1979;206:847-50.

3 Lüders HO. The supplementary sensorimotor area: an overview. Adv Neurol 1996;70:1-16.

4 Krainik A, Lehéricy S, Duffau $\mathrm{H}$, et al. Role of the supplementary motor area in motor deficit following medial frontal lobe surgery. Neurology 2001;57:871-8.

5 Crosson B, Sadek JR, Maron L, et al. Relative shift in activity from medial to lateral frontal cortex during internally versus externally guided word generation. J Cogn Neurosci 2001;13:272-83.

6 Krainik A, Lehéricy $S$, Duffau $H$, et al. Postoperative speech disorder after medial frontal surgery: role of the supplementary motor area. Neurology 2003;60:587-94.

7 Duffau H, Capelle L. Preferential brain locations of low-grade gliomas. Cancer 2004;100:2622-6.

8 Chassagnon S, Minotti L, Kremer S, et al. Somatosensory, motor, and reaching/ grasping responses to direct electrical stimulation of the human cingulate motor areas. J Neurosurg 2008;109:593-604.

9 Laplane D, Talairach J, Meininger V, et al. Clinical consequences of corticectomies involving the supplementary motor area in man. J Neurol Sci 1977;34:301-14

10 Fontaine D, Capelle L, Duffau H. Somatotopy of the supplementary motor area: evidence from correlation of the extent of surgical resection with the clinical patterns of deficit. Neurosurgery 2002;50:297-303.

11 Martino J, Gabarrós A, Deus J, et al. Intrasurgical mapping of complex motor function in the superior frontal gyrus. Neuroscience 2011;179:131-42.

12 Luppino G, Matelli M, Camarda R, et al. Corticocortical connections of area F3 (SMA-proper) and area F6 (pre-SMA) in the macaque monkey. J Comp Neurol 1993;338:114-40.

13 Rizzolatti G, Luppino G, Matelli M. The classic supplementary motor area is formed by two independent areas. Adv Neurol 1996;70:45-56.

14 Schmahmann JD, Pandya DN. Fiber pathways of the brain. Oxford: Oxford University Press, 2009.

15 Vergani F, Morris CM, Mitchell P, et al. Raymond de Vieussens and his contribution to the study of white matter anatomy. J Neurosurg 2012;117:1070-5.

16 Klingler J. Erleichterung der makroskopischen Präparation des Gehirns durch den Gefrierprozess. Schweiz Arch Neur Psychiat 1935:36:247-56. 
17 Türe U, Yaşargil MG, Friedman $\mathrm{AH}$, et al. Fiber dissection technique: lateral aspect of the brain. Neurosurgery 2000;47:417-26.

18 Martino J, De Witt Hamer PC, Vergani F, et al. Cortex-sparing fiber dissection: an improved method for the study of white matter anatomy in the human brain. J Anat 2011:219:531-41.

19 Jones DK, Simmons A, Williams SCR, et al. Non-invasive assessment of axonal fiber connectivity in the human brain via diffusion tensor MRI. Magn Reson Med 1999:42:37-41.

20 Lehéricy S, Ducros M, Krainik A, et al. 3-D diffusion tensor axonal tracking shows distinct SMA and pre-SMA projections to the human striatum. Cereb Cortex 2004; 14:1302-9

21 Klein JC, Behrens TE, Robson MD, et al. Connectivity-based parcellation of human cortex using diffusion MRI: Establishing reproducibility, validity and observer independence in BA 44/45 and SMA/pre-SMA. Neuroimage 2007:34:204-11.

22 Oishi K, Zilles K, Amunts K, et al. Human brain white matter atlas: identification and assignment of common anatomical structures in superficial white matter. Neuroimage 2008;43:447-57.

23 Ford A, McGregor KM, Case K, et al. Structural connectivity of Broca's area and medial frontal cortex. Neuroimage 2010;52:1230-7.

24 Dell'Acqua F, Scifo P, Rizzo G, et al. A modified damped Richardson-Lucy algorithm to reduce isotropic background effects in spherical deconvolution. Neurolmage 2010;49:1446-58.

25 Dell'Acqua F, Simmons A, Williams SCR, et al. Can spherical deconvolution provide more information than fiber orientations? Hindrance modulated orientational anisotropy, a true-tract specific index to characterize white matter diffusion. Hum. Brain Mapp 2013;34:2464-83.

26 Talairach J, Bancaud J. The supplementary motor area in man (anatomofunctional findings by stereo-electroencephalography in epilepsy). Int J Neurol 1966;5: 330-47.

27 Rostomily RC, Berger MS, Ojemann GA, et al. Postoperative deficits and functional recovery following removal of tumors involving the dominant hemisphere supplementary motor area. J Neurosurg 1991;75:62-8.

28 Penfield W, Rasmussen R. The cerebral cortex of man. New York: The Macmillan Company, 1950.
29 Ikeda A, Yazawa S, Kunieda T, et al. Cognitive motor control in human pre-supplementary motor area studied by subdural recording of discrimination/ selection-related potentials. Brain 1999:122:915-31.

30 Catani M, Dell'acqua F, Vergani F, et al. Short frontal lobe connections of the human brain. Cortex 2012:48:273-91.

31 Alexander GE, Crutcher MD. Functional architecture of basal ganglia circuits: neural substrates of parallel processing. Trends Neurosci 1990;13:266-71.

32 Lehericy $\mathrm{S}$, Duffau $\mathrm{H}$, Cornu $\mathrm{P}$, et al. Correspondence between functional magnetic resonance imaging somatotopy and individual brain anatomy of the central region: comparison with intraoperative stimulation in patients with brain tumors. J Neurosurg 2000;92:589-98

33 Kinoshita M, Shinohara $\mathrm{H}$, Hori $\mathrm{O}$, et al. Association fibers connecting the Broca center and the lateral superior frontal gyrus: a microsurgical and tractographic anatomy. J Neurosurg 2012:116:323-30.

34 Catani M, Mesulam MM, Jakobsen $\mathrm{E}$, et al. A novel frontal pathway underlies verbal fluency in primary progressive aphasia. Brain 2013;136:2619-28.

35 Martino J, de Lucas EM, Ibáñez-Plágaro FJ, et al. Foix-Chavany-Marie syndrome caused by a disconnection between the right pars opercularis of the inferior frontal gyrus and the supplementary motor area. J Neurosurg 2012;117:844-50.

36 Morecraft RJ, Van Hoesen GW. Cingulate input to the primary and supplementary motor cortices in the rhesus monkey: evidence for somatotopy in areas $24 \mathrm{C}$ and 23c. J Comp Neurol 1992;322:471-89.

37 Northoff G, Richter A, Gessner M, et al. Functional dissociation between media and lateral prefrontal cortical spatiotemporal activation in negative and positive emotions: a combined fMRI/MEG study. Cereb Cortex 2000;10:93-107.

38 Oliveri M, Babiloni C, Filippi MM, et al. Influence of the supplementary motor area on primary motor cortex excitability during movements triggered by neutral or emotionally unpleasant visual cues. Exp Brain Res 2003;149:214-21.

39 Russell SM, Kelly PJ. Incidence and clinical evolution of postoperative deficits after volumetric stereotactic resection of glial neoplasms involving the supplementary motor area. Neurosurgery 2007;61(1 Suppl):358-67.

40 Bizzi $A$, Nava $S$, Ferré $F$, et al. Aphasia induced by gliomas growing in the ventrolateral frontal region: assessment with diffusion MR tractography, functional MR imaging and neuropsychology. Cortex 2012;48:255-72. 